\title{
Long-term Increases in the Evoked Population Spike in the CA1 Region of Rat Hippocampus Induced by $\beta$-Adrenergic Receptor Activation
}

\author{
Lise R. Heginbotham ${ }^{1}$ and Thomas V. Dunwiddie ${ }^{2,3}$ \\ 'Program in Neuroscience, Harvard Medical School, Boston, Massachusetts 02115, '2Department of Pharmacology, \\ University of Colorado Health Sciences Center, Denver, Colorado 80262, and ${ }^{3}$ Veterans Administration Medical Research \\ Service, Denver, Colorado 80220
}

The effects of the selective $\beta$-adrenergic receptor agonist isoproterenol (ISO) were characterized in the CA1 region of the rat hippocampal slice preparation. As has been previously described, $500 \mathrm{~nm}$ ISO increased the amplitude of the evoked population spike response without having any effect upon field EPSP (fEPSP) responses. However, the increase in the population spike response was quite persistent and was not reversed by $>\mathbf{3 0}$ min of washout in the majority of the slices tested; we have termed this prolonged increase $\beta$-adrenergic potentiation (BAP). As with the acute effect of ISO, BAP is confined to an increase in the population spike response and not the fEPSP. In input-output curves, this was clearly observed as a persistent leftward shift in the EPSP-population spike relationship. Similar long-term increases in the population spike could also be elicited by superfusion of the slices for 10 min with 20-25 $\mu \mathrm{M}$ norepinephrine (NE). Although both the acute and the long-term effects of ISO were blocked by pretreatment with timolol, a $\beta$-adrenergic antagonist, the long-term effects were not reversed by superfusion with timolol following ISO treatment, demonstrating that the prolonged effects were not due to slow washout of ISO from the tissue. BAP was not blocked by pretreatment with $50 \mu \mathrm{M}$ 2-amino-5-phosphonovaleric acid, an NMDA receptor antagonist that blocks hippocampal longterm potentiation and the long-lasting changes in synaptic responses induced in the dentate gyrus by NE and ISO. These experiments demonstrate that NE can induce a novel and long-lasting increase in the excitability of the pyramidal neurons of the CA1 region following relatively brief activation of $\beta$-adrenergic receptors, and that this response is distinct from the other long-lasting effects that have been described in other regions.

Norepinephrine (NE) has been reported to have widely varying effects upon neural activity, including both inhibitory and excitatory effects upon single-unit activity and evoked responses (Segal and Bloom, 1974a,b; Woodward et al., 1979; Mueller et al., 1981; Segal, 1982; Basile and Dunwiddie, 1984) and poten-

Received Dec. 19, 1990; revised Mar. 14, 1991; accepted Mar. 22, 1991.

This research was supported by National Institute on Drug Abuse Grant DA 02702 and by the Veterans Administration Medical Research Service.

Correspondence should be addressed to Thomas V. Dunwiddie, Department of Pharmacology C236, University of Colorado Health Sciences Center, 4200 East 9 th Avenue, Denver, CO 80262.

Copyright (c) 1991 Society for Neuroscience 0270-6474/91/112519-09\$03.00/0 tiation of responses to inhibitory and excitatory neurotransmitters (Moises et al., 1979; Woodward et al., 1979; Rogawski and Aghajanian, 1980; Waterhouse et al., 1988). In the CAl region of the rat hippocampus, we have shown that NE can either potentiate or attenuate an evoked population spike response in a concentration-dependent manner, while having no effect upon the field EPSP (fEPSP) response, suggesting a postsynaptic locus of action. Low concentrations of NE increase the excitability of the CAl pyramidal neurons through actions upon $\beta_{1}$-adrenergic receptors (Mueller et al., 1981, 1982a,b; Fowler and O'Donnell, 1988), which results in a potentiation of the evoked CA1 population spike. At higher concentrations of NE, $\alpha_{1}$-adrenergic receptor-mediated attenuation of the population spike is observed (Mueller et al., 1981, 1982a,b; Mynlieff and Dunwiddie, 1988). The fact that both stimulation of the locus coeruleus (Smith et al., 1989; Washburn and Moises, 1989), which provides the noradrenergic innervation of the hippocampus, and application of indirect-acting sympathomimetics to the hippocampal slice (Mueller et al., 1982a) produce increases in the population spike response suggests that the $\beta$-receptor-mediated phenomenon may be the more physiologically relevant of the two actions.

In the dentate gyrus of the hippocampal formation, NE has been reported to increase not only the population spike evoked by perforant path stimulation, but the fEPSP, as well (Lacaille and Harley, 1985; Stanton and Sarvey, 1987), suggesting a possible presynaptic locus of action, and these effects appear to be mediated by $\beta$-receptors (Lacaille and Harley, 1985). What is most striking about the response in the dentate gyrus is the fact that it is very long lasting (Neuman and Harley, 1983; Lacaille and Harley, 1985; Stanton and Sarvey, 1985a; Stanton et al., 1989); in experiments in the intact rat, the response can be maintained at potentiated levels for a number of hours (Neuman and Harley, 1983). Because NE affects the fEPSP in the dentate but not the $\mathrm{CAl}$ region, it seems likely that the responses to $\mathrm{NE}$ seen in these two regions arise through distinct mechanisms.

While there are a number of studies that suggest that the responses in the dentate gyrus are quite persistent, most reports of adrenergic responses in the $\mathrm{CA} 1$ region have focused on the short-term effects of NE on evoked responses. For the most part, the effects of NE show relatively good reversibility upon washing (Mueller et al., 1981; Madison and Nicoll, 1982, 1986a,b; Haas and Konnerth, 1983), though there is a suggestion that some of the effects in the CA1 region can be more persistent (Anwyl and Rowan, 1984). The purpose of the present study 
was to examine in greater detail the $\beta$-receptor-mediated potentiation of the CA1 population spike, and to characterize the persistence of this response. In these experiments, we show that stimulation of $\beta$-receptors, either by the $\beta$-receptor-selective agonist isoproterenol (ISO), or by NE itself, results in a potentiation of the CAl population spike response that can last for as long as several hours.

\section{Materials and Methods}

Chemicals and drugs. An artificial cerebrospinal fluid (aCSF) was used in all experiments. The final concentration of the buffer was $124 \mathrm{~mm}$ $\mathrm{NaCl}, 25.7 \mathrm{~mm} \mathrm{NaHCO}, 10 \mathrm{~mm} \alpha$-glucose, $3.3 \mathrm{~mm} \mathrm{KCl}, 2.4 \mathrm{~mm}$ $\mathrm{MgSO}_{4}, 2.5 \mathrm{mM} \mathrm{CaCl}_{2}$, and $1.2 \mathrm{mM} \mathrm{KH}_{2} \mathrm{PO}_{4}$; all chemicals were obtained from Sigma or from J. T. Baker (Phillipsburg, NJ). After saturating the medium with $95 \% \mathrm{O}_{2} / 5 \% \mathrm{CO}_{2}$, the $\mathrm{pH}$ of the aCSF was 7.3-7.4. L-Isoproterenol, theophylline, reserpine, and D,L- $\alpha$-methyl-p-tyrosine were from Sigma Chemical Co. Timolol maleate was obtained from Merck, Sharp, and Dohme. Drugs used on the hippocampal slices were dissolved in deionized water in at least $100 \times$ the final concentration desired. The drugs were then added to the superfusate with Razel syringe pumps. A previous study has shown that dilution of the medium by this amount has no effect on responses evoked in the slices (Mueller et al., 1981).

Tissue preparation. Male Sprague-Dawley rats (150-200 gm, obtained from Sasco, Omaha, NE) were used in this study. Following decapitation, the brain was removed and placed in a cup of oxygenated, icecold aCSF. The hippocampus was dissected free of the rest of the brain, placed on the stage of a Sorvall TC-2 Tissue Sectioner with its longitudinal axis perpendicular to the blade, and cut into $400-\mu \mathrm{m}$ slices. The slices were lifted from the blade with a paintbrush and placed in a Petri dish containing oxygenated, ice-cold aCSF. The slices were then transferred to a recording chamber, where they were placed on a nylon net at a gas-liquid interface. The $\mathrm{O}_{2} / \mathrm{CO}_{2}$ gas mixture was humidified and then directed into the chamber above the slices. The slices incubated at $34^{\circ} \mathrm{C}$ for at least $1 \mathrm{hr}$ before recording started.

Electrophysiological recording. Before recording, slices were superfused at a rate of $2-4 \mathrm{ml} / \mathrm{min}$ with oxygenated aCSF warmed to 33$34^{\circ} \mathrm{C}$. In most experiments, recordings were made simultaneously from pairs of slices in a single recording chamber. Stimulating and recording electrodes were placed in the Schaffer collateral pathway and in the C.A 1 cell layer, respectively, under visual guidance. Stimulating pulses of 0.2 msec duration were delivered through bipolar stimulating electrodes, made from Formvar-insulated nichrome wire (A-M Systems, Inc.). The stimulation intensity was set so that the amplitude of the population spike was equal to the amplitude of the positive-going EPSP response in the cell body layer (usually $1-3 \mathrm{mV}$; see Fig. $7 B, D$ ). Unless otherwise noted, stimuli were delivered at a rate of once every $30 \mathrm{sec}$. When paired pulses were used, two pulses, both of equal intensity and 0.2 -msec duration, were delivered with an interpulse interval of $20 \mathrm{msec}$. Longterm potentiation (LTP) was elicited by delivering a train of pulses at $100 \mathrm{~Hz}$ for $1 \mathrm{sec}$.

Glass recording electrodes (Glass Co. of America, Inc.) were pulled on a micropipette puller (Industrial Science Associates, Inc.), bumped to obtain resistances of $1-3 \mathrm{M} \Omega$, and filled with $3 \mathrm{mM} \mathrm{NaCl}$. Recorded signals were passed through either a DAM 50 or a DAM 5-A differential amplifier (World Precision Instruments), displayed on a Gould type 1421 oscilloscope, and fed into an Epson Equity III $^{+}$computer for analysis and storage.

Data interpretation. The fEPSP was normally measured from potentials recorded in the pyramidal cell layer and was determined as the slope of the initial rising phase of the response. The height of the population spike was measured as the length of a vertical line, drawn from the minimum of the population spike to the line that joined the two positive peaks of the field response. In some experiments, fEPSPs were recorded in stratum radiatum; in these experiments, the amplitude of the potential was measured at a fixed latency on the falling phase of the response, chosen to be well before any population spike-induced perturbations in this response.

In all experiments, each drug application was preceded by a control period of at least $10 \mathrm{~min}$. Isoproterenol application was for $10 \mathrm{~min}$ and was followed by a $30-\mathrm{min}$ wash period. Isoproterenol-induced potentiation was judged to be long lasting if it met an arbitrary $t$-test criterion, in which the $t$ value for the 10 responses taken immediately prior to
ISO treatment compared to the 10 responses recorded between 25 and $30 \mathrm{~min}$ of washout showed a $p<0.001$.

Comparisons of the effects of drug treatments (normalized as percent of control) between groups of slices were performed using a $t$ test for differences between two independent means. The effect of repeated drug applications to the same set of slices was compared using a paired $t$ test. In both cases, results were considered significant at $p<0.05$. Covariations between two parameters were established using the $\chi^{2}$ test, at $p$ $<0.05$. Linear regressions were performed using the least-squares method, and correlations were considered significant at $p<0.05$.

Norepinephrine depletions. In order to deplete norepinephrine stores, reserpine was administered to four animals $(0.5 \mathrm{mg} / \mathrm{kg}$, once per day for $3 \mathrm{~d}$, s.c.). The day the animals were to be used, a single injection of the competitive inhibitor of NE synthesis $\alpha$-methyl-p-tyrosine was given $1 \mathrm{hr}$ prior to decapitation $(200 \mathrm{mg} / \mathrm{kg}$, i.p.). The extent of depletion was not quantified, though we have previously shown this protocol to be effective in reducing hippocampal NE. Animals were judged to be NE depleted if spinal reflexes upon decapitation were not noticcable, and this was the case in all animals tested. We have previously observed that the loss of these spinal reflexes is an accurate indicator of $>90 \%$ depletion of NE (B. J. Hoffer and T. V. Dunwiddie, unpublished observations).

\section{Results}

Previous studies from our laboratory have established that perfusion of hippocampal slices with ISO results in a rapid increase in the CAl population spike, and that peak increases are observed at concentrations of approximately $500 \mathrm{nM}$ (Mueller et al., 1981). The present study confirmed these observations concerning the acute effects of ISO (Fig. 1). In our experiments, 500 $\mathrm{nM}$ ISO reliably (46 of 47 slices) elicited an increase in population spike amplitude (peak increase above control was 100.2 $\pm 6.7 \% ; n=47$ ). In 28 of 47 slices, the increase in the population spike response far outlasted the duration of superfusion with ISO (Fig. 1; see also Figs. 3-7). We refer to this persistent change in the response as $\beta$-adrenergic potentiation (BAP). Because NEinduced changes in the fEPSP have been described in the dentate gyrus, we characterized changes in the fEPSP in addition to the population spike response. As we have previously reported for the acute response to NE (Mueller et al., 1981), the fEPSP response (measured within the stratum pyramidale) was unaffected by ISO (mean change, $-2.7 \pm 1.4 \% ; n=36$; Fig. 1). The absence of an effect of ISO on the fEPSP was confirmed by recording simultaneously in stratum radiatum and stratum pyramidale (e.g., as in Figs. 1, 2); the mean increase in a group of four slices in the population spike response was $296 \pm 166 \%$, and the corresponding change in the fEPSP in the same group of slices was $0.4 \pm 1.9 \%$. Input-output curves also demonstrated significant changes in the population spike response that were not accompanied by parallel changes in the fEPSP (Fig. 2).

If the prolonged increase in the population spike response werc duc to a persistent $\beta$-adrencrgic response, then we predicted that responses to a second application of ISO should be reduced. In general, the magnitude of the acute response to a second application of ISO was significantly less than the response to the first application (e.g., Fig. 3A). In a group of slices tested with protocols similar to that in Figure $3 A$ (washout times following ISO varied from 30 to $90 \mathrm{~min}$ ), a second application of ISO elicited an approximately 10 -fold smaller acute response $(12.5 \pm 3.8 \%$ increase above control; $n=5)$ than did the first application (111 $\pm 41.6 \%$ increase; $n=5 ; p<0.05$, paired $t$ test). Because the second response might have been smaller because of a ceiling effect for the population spike, we conducted a second series of experiments in which the stimulus intensity was reduced after the $30-\mathrm{min}$ wash to evoke a population spike 


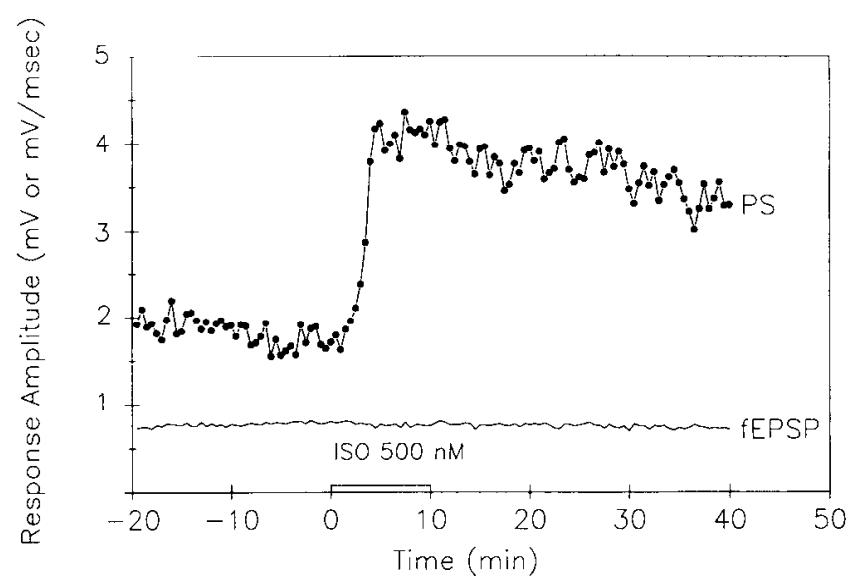

Figure 1. Effect of ISO on the population spike and fEPSP amplitude in a single slice. The population spike and fEPSP were recorded simultaneously with separate electrodes located in the stratum pyramidale and stratum radiatum, respectively. The slice was superfused with 500 nм ISO from 0 to $10 \mathrm{~min}$, as denoted by the horizontal line along the $x$-axis, and produced an approximately twofold increase of the population spike amplitude ( $P S$; indicated by line with solid circles). No concurrent change in fEPSP was noted. This slice met our criterion for BAP, which was an elevated population spike response following 30 min of washout.

similar in magnitude to that prior to the initial ISO application (Fig. $3 B$ ). In these slices, as well, the acute effect of a second treatment with ISO was significantly less than the response to the original treatment $(87 \pm 23, n=14$, vs. $104 \pm 28, n=14$; $p<0.05$, paired $t$ test). Because of this reduced sensitivity, slices were exposed to ISO only once in the rest of the experiments.

One possible explanation for the persistent elevation of the population spike response could be a slow washout of the agonist (ISO) from the slice. If our chamber had ideal mixing of fresh medium with the bath, an initial concentration of $500 \mathrm{~nm}$ ISO would be reduced to $10 \mathrm{~nm}$ (the threshold for ISO responses) in less than $3 \mathrm{~min}$, and below $1 \mathrm{pm}$ in $10 \mathrm{~min}$. However, because the mixing of drug is probably not ideal, we used a direct approach to demonstrate that the responses did not persist because of the continued presence of agonist. Because the acute response to ISO is mediated by a $\beta_{1}$-adrenoceptor (Mueller et al., 1981; Fowler and O'Donnell, 1988), and because it seemed likely that the long-lasting increase in the population spike amplitude was also mediated by this receptor, we tested the ability of the $\beta$-adrenergic receptor antagonist timolol to block both the acute and long-term effects of ISO. Furthermore, we investigated the ability of this antagonist to reverse BAP when administered during the washout period (Fig. 4). Both the short-term and the long-term increase in the population spike response elicited by ISO were completely blocked by pretreating the slices with 1 $\mu \mathrm{M}$ timolol. Thus, the long-lasting increase in the response seemed to be specifically induced by $\beta$-receptor activation.

However, BAP was completely unaffected by superfusion with $1 \mu \mathrm{M}$ timolol beginning $10 \mathrm{~min}$ after the onset of ISO treatment (Fig. 4). This suggests that BAP is not just the result of a continued activation of $\beta$-receptors by the agonist, but rather that there is a process occurring that, once initiated, is no longer sensitive to disruption by $\beta$-adrenergic receptor antagonists. As a further test of this hypothesis, we also characterized the effects of superfusion with NE $(20 \mu \mathrm{M})$ on the population spike response. Unlike ISO, NE is a substrate for reuptake (see Yasuda

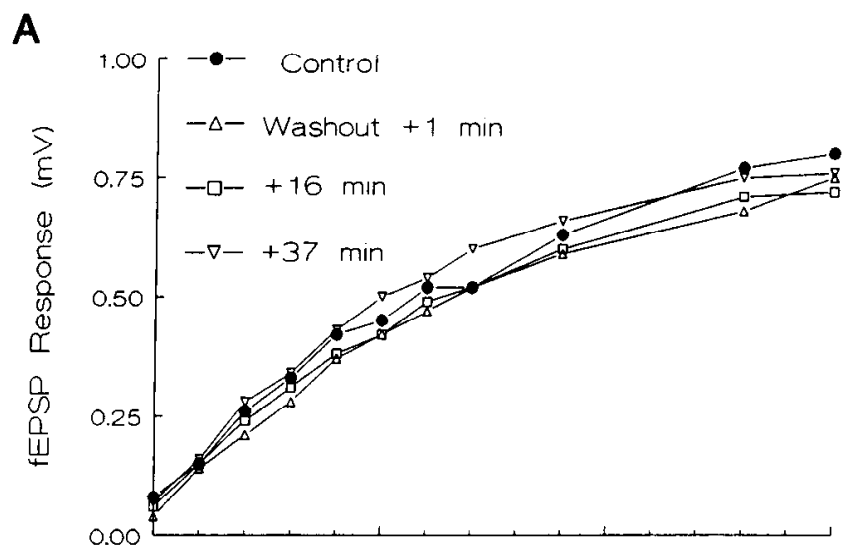

B

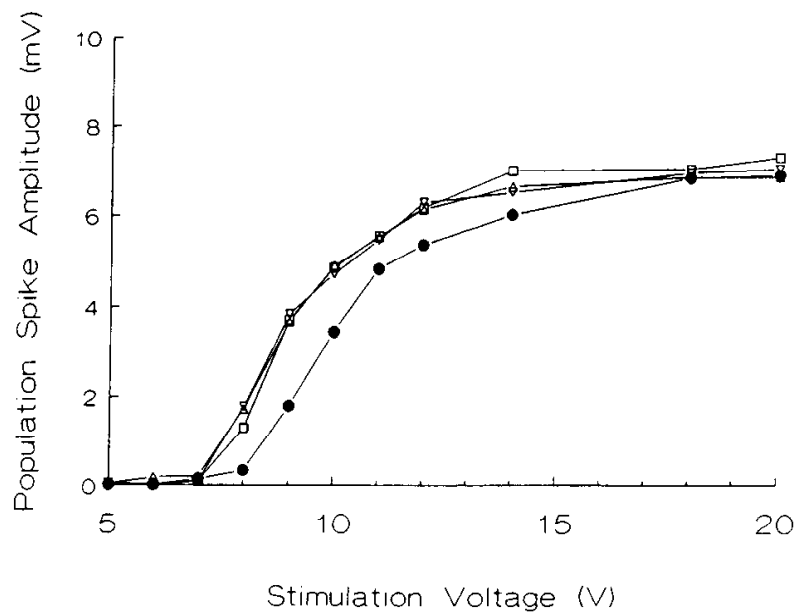

Figure 2. The effect of ISO on input-output curves. $A$ illustrates the amplitude of the evoked fEPSP as a function of stimulation intensity (same stimulation voltage scale as in $B$ ). The fEPSP was recorded in stratum radiatum and was measured by determining the difference between the response amplitude at 4.02 and $4.86 \mathrm{msec}$ following the stimulus; these points were chosen to be after the presynaptic fiber spike, and before the distortion of the fEPSP introduced by the population spike that was evoked at high stimulation intensities. Input-output curves were determined prior to superfusion with $500 \mathrm{nM}$ ISO, and beginning at 1,16 , and $37 \mathrm{~min}$ after the onset of washout. Each input-output curve took $4 \mathrm{~min}$ to acquire. $B$ illustrates the input-output curve for the population spike response recorded from the same slice in stratum pyramidale; each of the curves obtained following ISO was markedly shifted to the left, but there did not appear to be a significant change in the maximal response. The increase in the population spike response at intermediate stimulation intensities was maintained in this experiment for at least 37 min of washout with no apparent decrement.

et al., 1984, and references therein), and we have shown directly with electrochemical measurements that it is cleared quite rapidly from hippocampal slices $\left(t_{1 / 2}=30 \mathrm{sec}\right.$; Su et al, 1990). In slices superfused for $10 \mathrm{~min}$ with $20 \mu \mathrm{M} \mathrm{NE}$, the population spike response remained significantly potentiated $(38 \pm 5.1 \%$ above control levels; $N=10$ slices) $30 \mathrm{~min}$ following the termination of superfusion. BAP could also be elicited in slices pretreated with the $\alpha$-adrenergic receptor antagonist phentolamine $(10 \mu \mathrm{M})$ for $>30$ min prior to superfusion with $N E$ (data not shown), again suggesting the involvement of a $\beta$-adrenergic receptor.

It has been suggested in a number of studies that the persistent effects of NE or ISO in the dentate gyrus depend upon the activation of NMDA receptors, because they can be blocked 
A

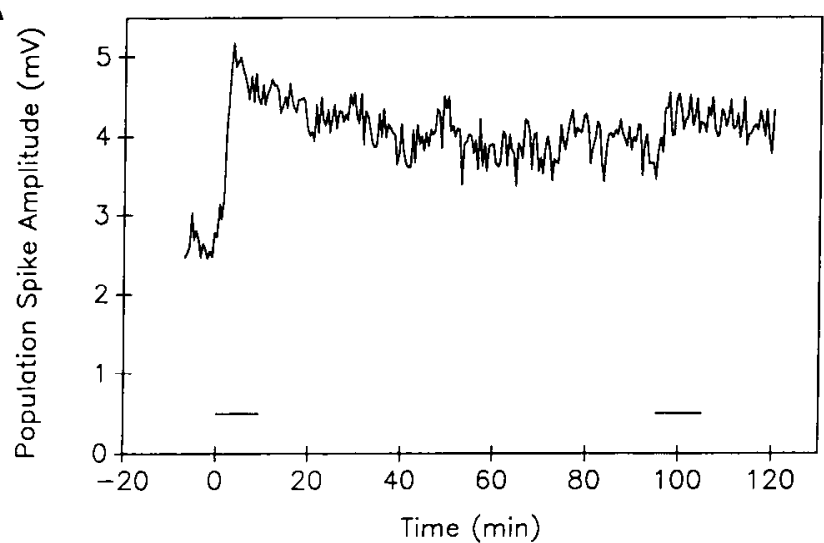

B

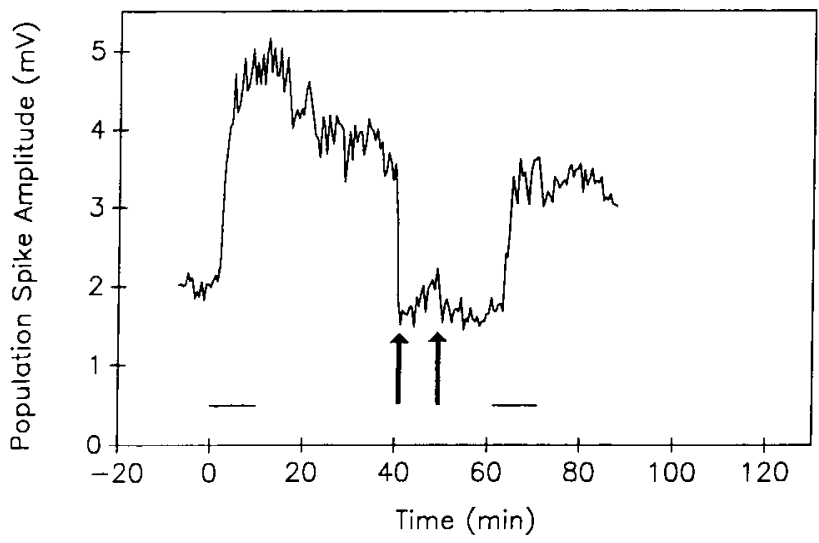

Figure 3. Effect of repeated application of ISO. The slice in $A$ showed BAP for nearly $90 \mathrm{~min}$ following an initial application of $500 \mathrm{nM}$ ISO $(0-10 \mathrm{~min})$; the horizontal lines indicate the period of superfusion with ISO. A second application of ISO to this slice had only a marginal effect on the population spike amplitude. Increasing the stimulation intensity at the end of the experiment (not shown) markedly increased the population spike response, demonstrating that the response amplitude had not been maximal. $B$, A separate slice was treated with 500 nм ISO for $10 \mathrm{~min}$ and superfused with control medium for $30 \mathrm{~min}$; then, the stimulation voltage was reduced (arrows), and ISO was applied again $60 \mathrm{~min}$ following the initial treatment. In this case, the response to ISO was reduced but not blocked.

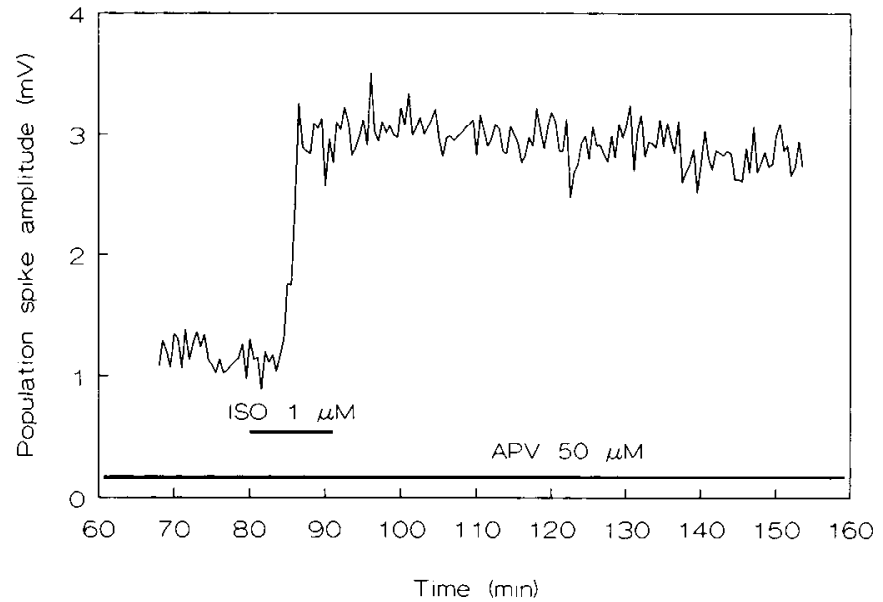

Figure 5. Effect of APV on ISO responses. This slice was superfused with $50 \mu \mathrm{M}$ APV for $75 \mathrm{~min}$ prior to and during a 10-min superfusion with $1 \mu \mathrm{M}$ ISO. The effect of ISO in this particular slice was, if anything, more long lasting than was normally observed in control slices. A similar lack of sensitivity to APV was observed with slices superfused with 500 nM ISO as well.

with superfusion with 2-amino-5-phosphonovaleric acid (APV) or other NMDA receptor antagonists (Burgard et al., 1989; Stanton et al., 1989). We examined this possibility by determining whether BAP was antagonized by superfusion with $50 \mu \mathrm{M}$ APV. As can be seen from Figure 5, APV did not block either the short-term or the long-term effects of ISO on the population spike amplitude. Significant acute increases in the population spike amplitude were observed in 14 of 14 slices tested with either $500 \mathrm{nM}$ or $1 \mu \mathrm{M}$ ISO in the presence of $50 \mu \mathrm{M}$ APV; BAP was observed in 7 of 10 slices tested with $500 \mathrm{nM}$ and three of four slices tested with $1 \mu \mathrm{M}$ ISO. A $100-\mathrm{Hz}, 1-\mathrm{sec}$ stimulation train failed to evoke significant LTP in any of four slices pretreated with $50 \mu \mathrm{M} \mathrm{APV}$, demonstrating that the concentration of APV used in these experiments was effective in blocking NMDA receptors, which are required for the initiation of LTP.
Figure 4. Effect of timolol on BAP. The mean \pm SEM population spike amplitude is displayed for each time point for slices run under three different protocols. Each point represents the averaged response, normalized to a baseline of $100 \%$, for four slices from three animals. In each case, $500 \mathrm{~nm}$ ISO was perfused from 0 to $10 \mathrm{~min}$, as noted by the horizontal line. A 10-min treatment with ISO resulted in a twofold enhancement, which persisted through a 30-min washout period (diamonds). Posttreatment with $1 \mu \mathrm{M}$ timolol after BAP onset (from 10 to $20 \mathrm{~min}$ ) had no effect on BAP (triangles), while pretreatment of the slices with $1 \mu \mathrm{M}$ timolol (from -10 to $10 \mathrm{~min}$ ) completely blocked the acute increase in the population spike as well as BAP (squares).

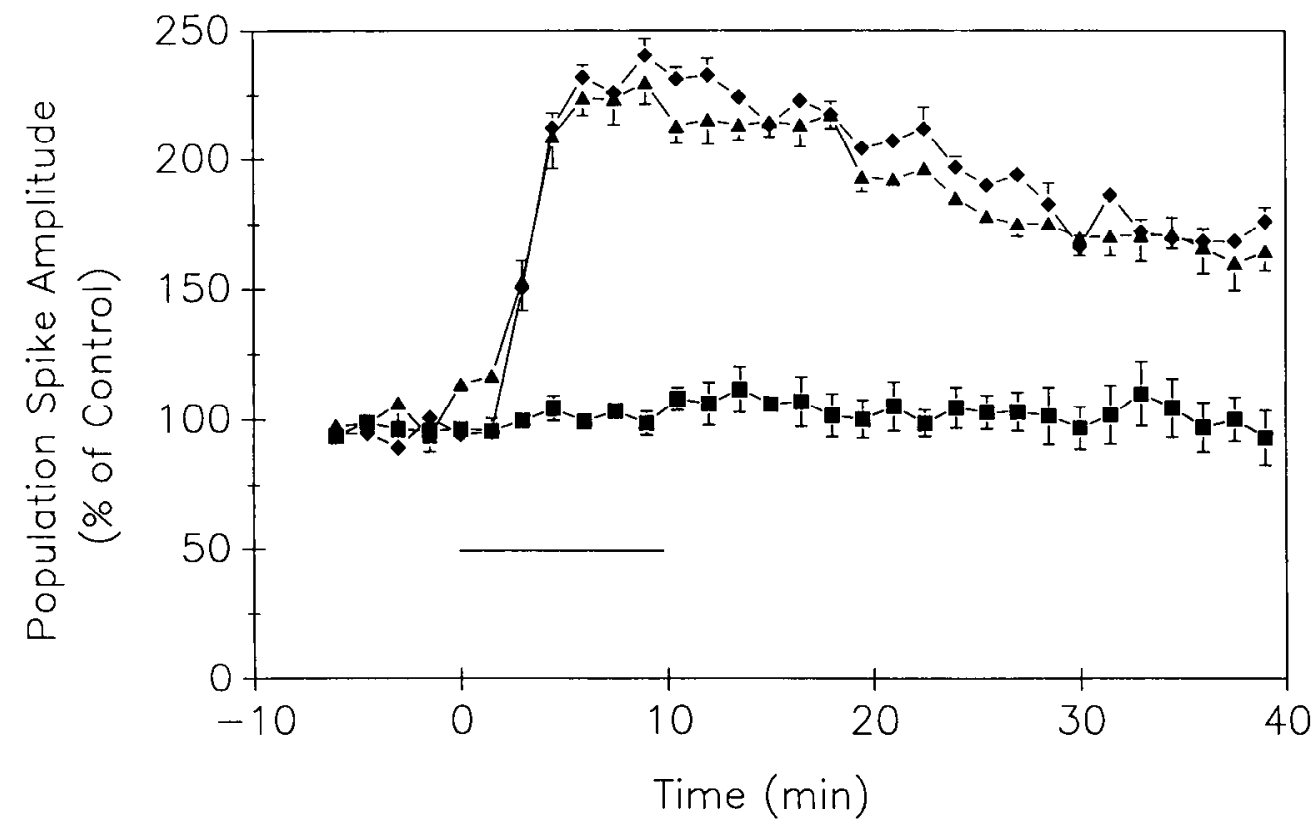



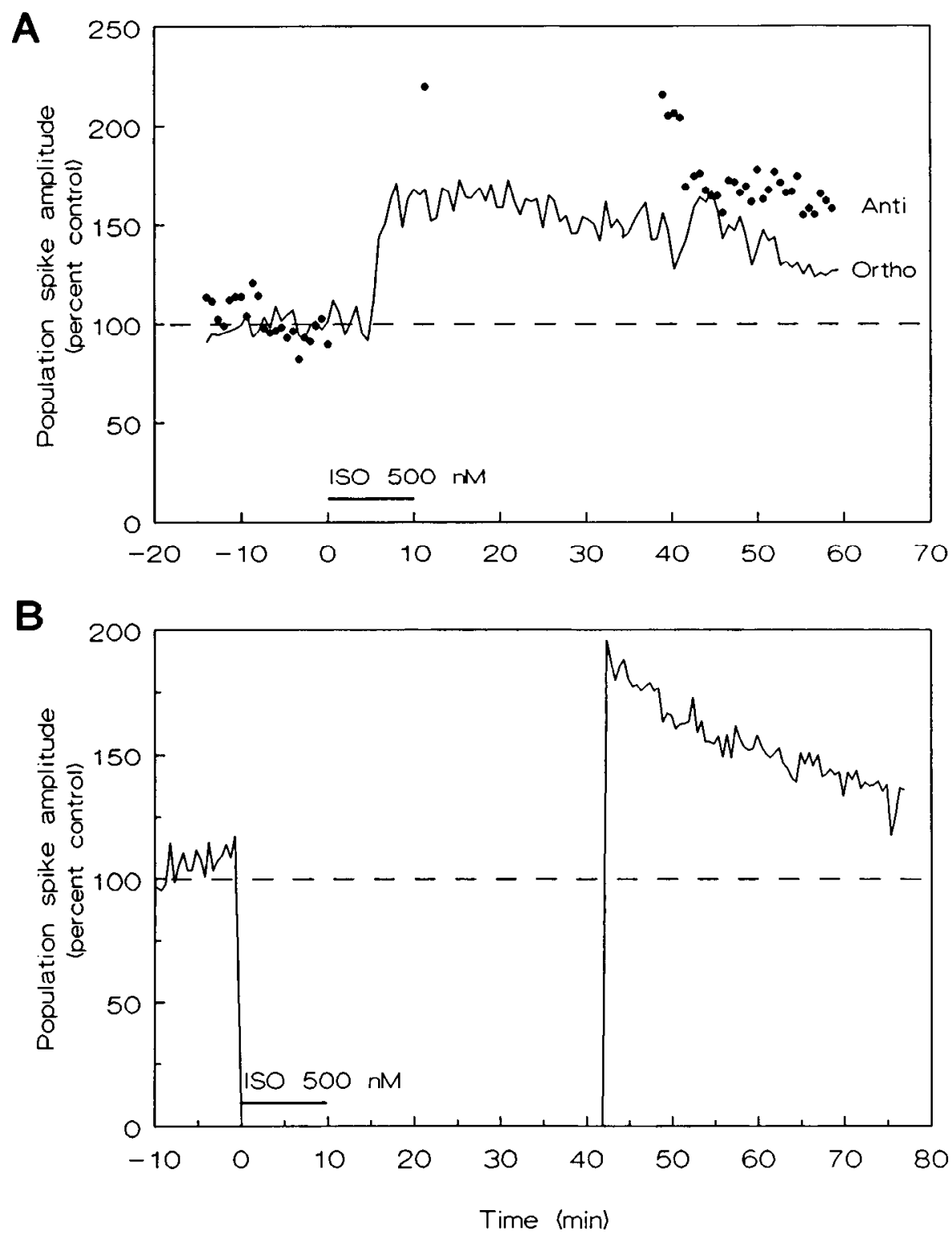

Figure 6. Effect of synaptic stimulation during ISO superfusion on BAP. In $A$, a single slice was stimulated alternately via normal orthodromic activation of the Schaffer collateral and commissural fibers (Ortho, line), and via antidromic activation of a different population of fibers synapsing in the same region (Anti, circles; see Dunwiddie and Lynch, 1978). The antidromic response was not stimulated at all during ISO superfusion, was stimulated once after $1 \mathrm{~min}$ of washout to demonstrate that the acute response to ISO had occurred, and then from $25 \mathrm{~min}$ to the end of the experiment. In $B$, the normal experimental protocol was used except that stimulation was stopped completely during ISO superfusion and for $32 \mathrm{~min}$ of washout. In both cases, normal BAP was observed.

Despite the fact that the stimulation rates used to test the pyramidal cell response were quite low $(0.033 \mathrm{~Hz})$, it seemed possible that the increase in the population spike response reflected an interaction between simultaneous $\beta$-adrenergic receptor activation and repetitive stimulation. To rule out this possibility, we conducted two types of experiments. In one protocol, we utilized two independent excitatory synaptic inputs to the CAl region (Dunwiddie and Lynch, 1978), one of which was stimulated at a rate of $0.033 \mathrm{~Hz}$ during the ISO superfusion, and the other was not. As can be seen in the example shown in Figure $6 \mathrm{~A}$, increases in the response occurred regardless of the stimulation condition. However, in this protocol, the pyramidal cells were still receiving stimulation during ISO superfusion, albeit via other synapses. Thereforc, two additional cxperiments were carried out in which stimulation was stopped completely during the ISO superfusion and the first $30 \mathrm{~min}$ of washout. Again, BAP occurred in the absence of stimulation (Fig. $6 \mathrm{~B}$ ).

Although the mean response from all ISO-treated slices was elevated at $30 \mathrm{~min}$ following ISO washout, there were individual slices that clearly did not show this effect. Two examples illustrating the variability in this response are shown in Figure 7. In the majority of slices tested ( 28 of 47 ), the population spike showed little or no decrement from the ISO-induced potentiation within the 30-min wash period, as seen in Figure $7 A$, and were still significantly potentiated at $30 \mathrm{~min}$, whereas in the remainder (19 of 47), the population spike amplitude returned to baseline levels, usually within $15-30 \mathrm{~min}$ (Fig. 7 B). This occurred despite the fact that nearly all slices ( 46 of 47 ) showed acute increases in the population spike response to ISO.

To quantitatively approach the issue of the duration of BAP, we characterized the rate of decay of the potentiated response during the period from 15 to $30 \mathrm{~min}$ after the beginning of washout. At this timc, washout of drug from the bath should be essentially complete, so that the rate of decline in the potentiated response should reflect the actual time course of BAP, rather than washout of the drug from the chamber. Figure 8 illustrates the rate of decay of the population spike during the latter half of the washout period for two individual slices and 
A

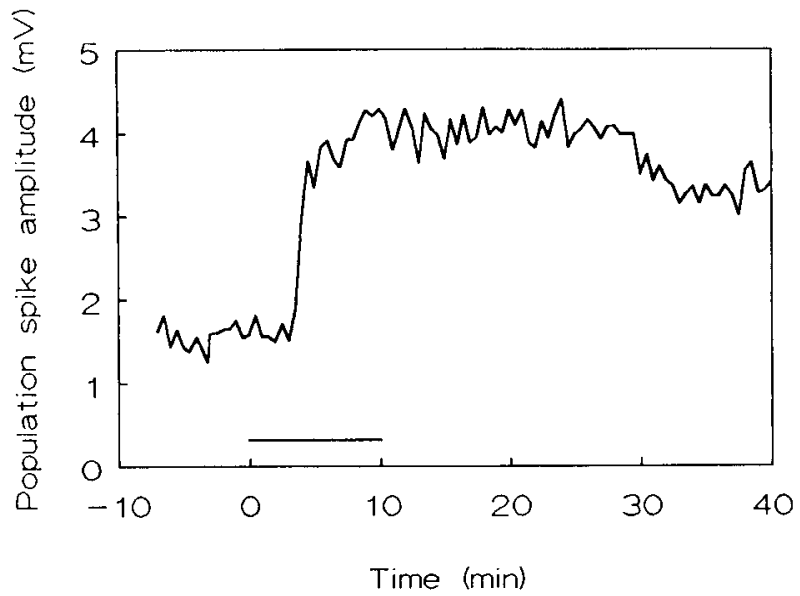

C

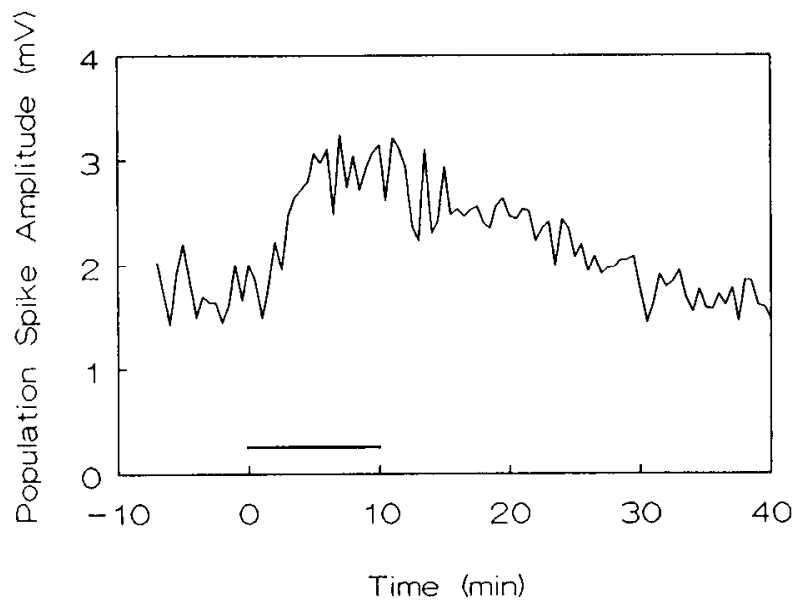

B

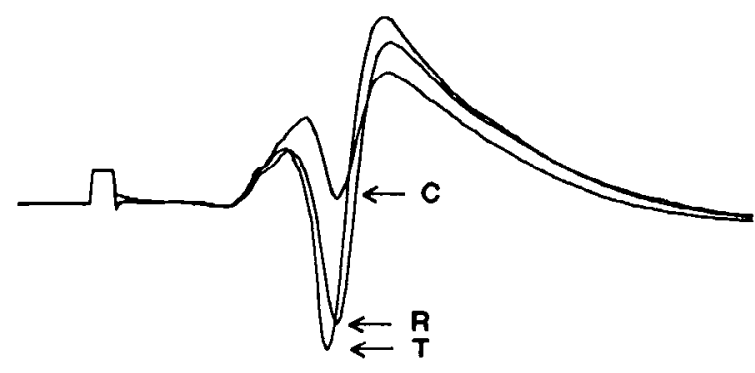

D

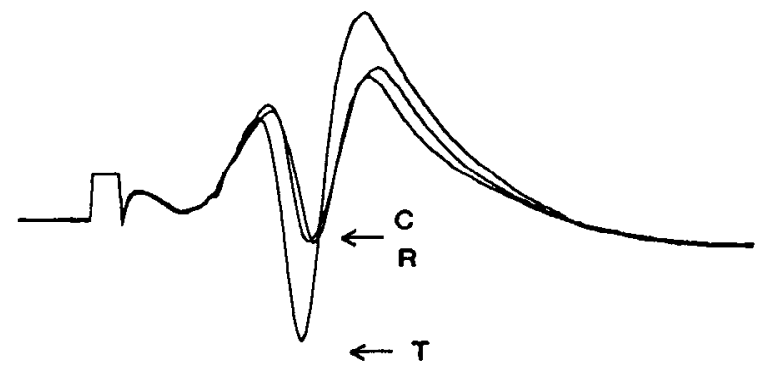

Figure 7. Variation in the duration of increased population spike responses following ISO treatment. ISO was superfused at $500 \mathrm{~nm}$ from 0 to $10 \mathrm{~min}$, as indicated by the horizontal lines in each panel. Each evoked response in $B$ and $D$ represents an average of 10 sweeps. The control period $(C)$ was averaged from -5 to $-0.0 \mathrm{~min}$; the ISO treatment period $(T)$, from 5 to $10 \mathrm{~min}$; and the recovery period $(R)$, from 35 to $40 \mathrm{~min}$. In both slices, ISO produced an approximately twofold increase in the population spike, while the slope of the initial positivity (the EPSP) was unaltered. Only the slice shown in $A$ and $B$ showed BAP. The duration of each trace was $20 \mathrm{msec}$, and the stimulus artifact is truncated to indicate $0.5 \mathrm{mV}$.

for the group of 24 slices that met our criterion for BAP. Although one cannot reliably estimate a $t_{1 / 2}$ from this $15-\mathrm{min}$ period of recovery, it is clear that, once established, BAP returns to baseline very slowly. If the response continued to decrease beyond $30 \mathrm{~min}$ in an exponential fashion at the same rate as in Figure 8 , this would correspond to a $t_{3 / 2}$ of $118 \mathrm{~min}(95 \% \mathrm{con}-$ fidence limits of $87.5-183 \mathrm{~min}$ ).

The variability in the duration of the potentiation led us to consider a variety of factors that might predict the duration of BAP in a given slice. Among the factors examined were (1) the magnitude of the acute response to ISO, (2) the magnitude of paired-pulse inhibition in a slice, (3) the ability of a slice to show LTP, and (4) the endogenous NE concentration of the slice. As far as the initial response to ISO was concerned, there was no significant difference in the initial increasc in the population spike between the group of slices that displayed BAP and those that did not (peak responses, $106 \pm 7.8 \%$ vs. $91.6 \pm$ $11.7 \%$, respectively; $p>0.1$ ). Paired-pulse inhibition can be used as a measure of the integrity of the inhibitory circuits within the hippocampus, and previous studies have demonstrated a significant correlation between the magnitude of paired-pulse inhibition and the effects of drugs that interact with inhibitory circuitry (Dunwiddie et al., 1980). In the present experiments, paired-pulse inhibition was defined as the ratio of the amplitude of the second population spike to the first when evoked with an interpulse interval of $20 \mathrm{msec}$. No correlation existed between this measure and the magnitude of BAP at $30 \mathrm{~min}\left(r^{2}=0.00\right.$; $n=8$ ), suggesting that local GABA-containing inhibitory circuits may not be involved in long-lasting BAP. LTP is another form of long-term plasticity induced by high-frequency stimulation. If there is some relationship between BAP and LTP, as has been suggested for LTP and the effects of NE in the dentate gyrus (Bliss et al., 1983; Stanton and Sarvey, 1985b, 1987), then one might observe an interaction between the magnitude of BAP and LTP in the same slice. However, the correlation between the magnitude of BAP and the magnitude of LIP that could be produced after the 30 -min washout from ISO was not statistically significant $\left(r^{2}=0.22 ; n=7\right)$.

We also hypothesized that, if large amounts of NE were being released following decapitation, this might affect the subsequent response of the slices to ISO. To determine if this was the case, NE was depleted with the storage inhibitor reserpine and the synthesis inhibitor $\alpha$-methyl-p-tyrosine. Slices from pretreated animals showed an incidence of long-lasting BAP (70\%, 7 of 10) that was similar to the control animals $(60 \%, 28$ of 47$)$, and there was no significant difference in the magnitude of either 


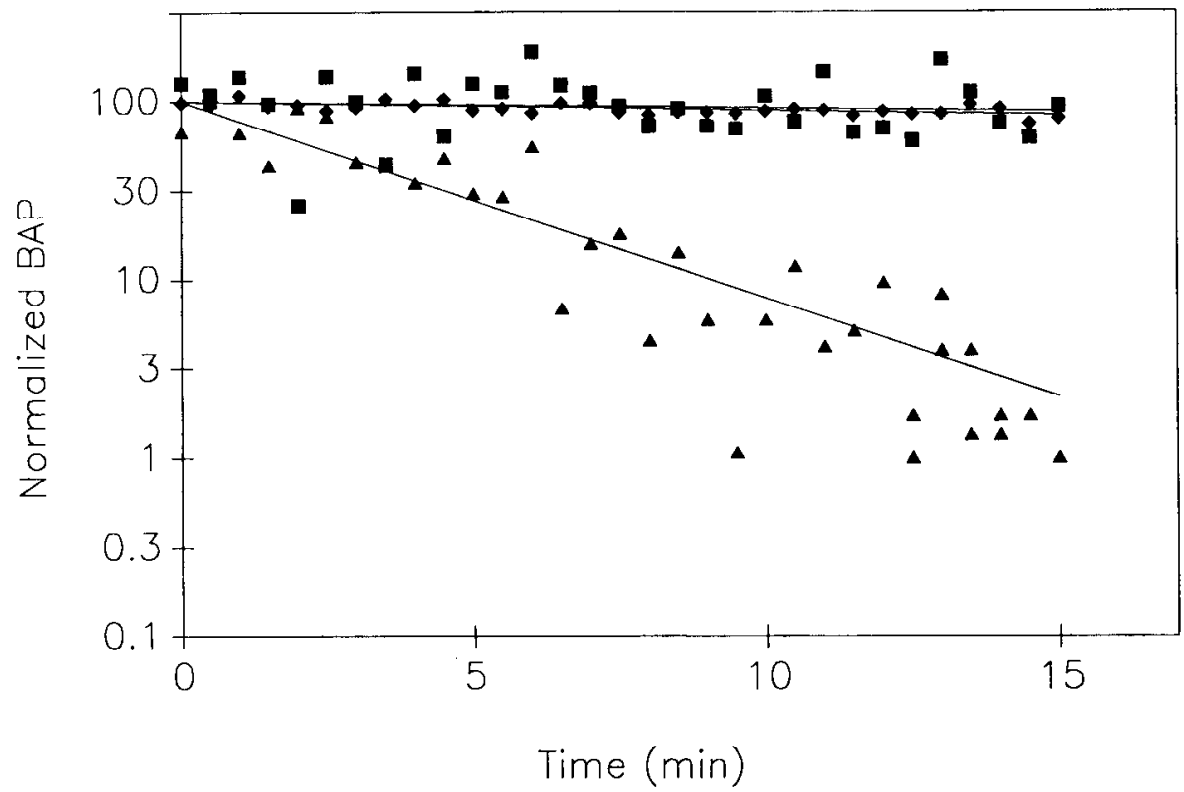

Figure 8. Variability in the rate of decline of BAP. The rate of decline of population spike amplitude is illustrated for the period from 15 to $30 \mathrm{~min}$ of washout for two single slices (triangles and squares), and for the average of 24 slices that showed BAP and for which we had data that included this time period (diamonds). Shown on a log scale on the ordinate is the magnitude of the enhancement above the pre-ISO baseline, expressed as percentage of the increase at $15 \mathrm{~min}$. One of the slices showed BAP (squares); the other did not (triangles). The lines were fit by a leastsquares criterion; correlation coefficients were -0.68 for the mean of 24 slices, -0.88 for the rapidly declining slice $\left(t_{1 / 2}\right.$ of $5.0 \mathrm{~min} ; 95 \%$ confidence limits of $7.8-11.6 \mathrm{~min}$ ), and -0.11 for the single slice with the slow decrement in BAP $\left(t_{1 / 2}\right.$ of $190 \mathrm{~min}$; confidence limits could not be determined because of the low $r$ value). the mean acute effect or the long-lasting effect of ISO between control and depleted animals $(p>0.05)$. Because there is a gradient along the septotemporal axis both in the number of $\beta$-adrenergic receptors and in NE levels (Loy et al., 1980; Kainbow et al., 1984), we investigated the possibility that the variability in BAP was related to the density of innervation. However, individual cases in which BAP either was or was not observed could be found regardless of the part of the hippocampus from which the slices were obtained.

\section{Discussion}

Previous studies have demonstrated that NE can induce longlasting potentiation (termed NE-LLP) of evoked responses in the dentate gyrus of the rat hippocampus. However, with the exception of a single report that suggested that washout of the effect of ISO in the CAl region was prolonged (Anwyl and Rowan, 1984), there have been no reports of persistent adrenergic responses in this region. The present study is the first to demonstrate that ISO and NE produce long-lasting changes in physiological responses in the CAl region. This persistent increase, which we refer to as BAP, consists of an increase in the population spike that is not accompanicd by any changes in the fEPSP response; in this respect, BAP is clearly distinct from NE-LLP. In the dentate, the primary effect is an increase in the fEPSP response, which suggests a presynaptic locus of the effect, whereas the changes in the $\mathrm{CA} 1$ region are clearly postsynaptic. Coupled with the fact that the response in the dentate gyrus is blocked by the NMDA receptor antagonist APV (Burgard et al., 1989), whereas BAP is unaffected by this agent, it is clear that different mechanisms must underlie these responses. Nevertheless, both types of potentiation appear to be mediated by $\beta$-adrenoreceptors and can be induced by NE, by the selective $\beta$-adrenergic agonist ISO, and by NE in the presence of the $\alpha$-adrenergic antagonist phentolamine.

The preceding extracellular recording studies do not shed much light upon the mechanism that underlies BAP. The main effect of ISO in the CAI region consists of an alteration of pyramidal ccll cxcitability, bccausc there was no change in the fEPSP response. A decrease in GABAergic inhibition might explain these effects, because GABA antagonists also increase the population spike with no effect on the fEPSP response. However, we found no correlation between the magnitude of BAP and the magnitude of paired-pulse inhibition, which is an indirect measure of the effectiveness of GABAergic inhibition in the slice. Although this is not a definitive test of this hypothesis, it suggests that inhibitory interneurons are not involved in BAP. An alternative mechanism underlying these changes would be either a prolonged depolarizing effect of ISO in CA1 pyramidal neurons, or a change in the threshold for spike generation. These potential mechanisms are considered explicitly in a following communication (Dunwiddie et al., 1991).

The reason for the persistence of the increase in the population spike response, the variability seen in BAP duration, and the apparent lack of any long-term effect in some slices is unclear. It is unlikely that the persistence of the effect reflects slow washout of drug from the tissue, because this would be relatively consistent and could not explain the variability that was observed. Furthermore, we frequently observed persistent and transient responses from each of a pair of slices that were recorded from simultaneously within a few hundred microns of each other in the recording chamber. This would appear to be highly unlikely if unsuccessful washout were the only explanation for our results. In addition, the rapid exchange of medium in the chamber and the fact that NE is rapidly taken up and removed from the extracellular space make this an unlikely possibility insofar as NE is concerned. The fact that second responses to ISO were significantly reduced also suggests that some aspect of cellular physiology has been persistently altered by the first exposure to ISO. Whether the smaller second response is the result of changes at the level of the $\beta$-receptor (e.g., desensitization) or at the effector level (perhaps the persistence of a phosphorylated substrate) remains unclear.

The reason for the variability in the long-term responses, despite the fact that nearly every slice responded to ISO with a robust short-term increase in the population spike, is another issue that remains unanswered. However, studies in the dentate gyrus have also reported considerable variability; in the study by Lacaille and Harley (1985), only $24 \%$ of the slices showed 
effects lasting longer than $30 \mathrm{~min}$. Although we tested a number of hypotheses concerning the duration of the response, involving such factors as the density of the innervation and the presence of endogenous NE, these experiments did not provide any indication as to what might predispose toward the long-lasting effect. It is possible that these differences might be related to the effects of other agents such as histamine or adenosine that, like ISO, can also affect cAMP levels in the hippocampal slice. Prior activation of such a system could modify subsequent ISO responses and alter the duration of BAP. Yet another possibility that has been suggested by others is that the production of longterm effects (at least in the dentate) may be partially dependent on the length of duration of exposure to ISO. Madison and Nicoll (1986a), who have not reported long-term effects on intracellularly recorded parameters in the CAl region, typically superfuse with $10 \mu \mathrm{M}$ NE for $<5 \mathrm{~min}$, whereas Stanton and Sarvey (1985a), who have reported perhaps the most consistent long-term effects of NE in dentate, use treatment with $50 \mu \mathrm{M}$ $\mathrm{NE}$ for $30 \mathrm{~min}$. However, we have generally avoided using these types of treatment protocols, because if continued exposure to high concentrations of agonist are required to consistently elicit BAP, this would makc its physiological relevance somewhat questionable.

Another issue concerns the relationship between BAP in the CAl region, and other long-lasting changes in excitability reported in the hippocampal formation. With LTP in the CAl and dentate gyrus (Bliss and Lomo, 1973; Schwartzkroin and Wester, 1975), and NE-LLP of the dentate gyrus (Stanton and Sarvey, 1987), an increase in the fEPSP has been reported that is concurrent with the increase in population spike, and is similarly long lasting. However, increases in the fEPSP response do not occur in the CAl region in response to ISO, demonstrating that BAP is not identical with any of these processes. Furthermore, APV blocks both NE-LLP and LTP in the dentate gyrus, but not BAP. Nevertheless, there are reasons to suppose that something very similar to BAP may occur in the dentate gyrus. First, there are reports that indicate that increases in the perforant path-evoked population spike may occur without any change in the fEPSP response (Neuman and Harley, 1983). Second, it has been reported that, even when changes do occur in the dentate fEPSP, they are of insufficient magnitude to account for the increase in the population spike (Lacaille and Harley, 1985); the same phenomenon has also been observed with LTP in the dentate (Bliss and Lomo, 1973). Finally, the recent observation of long-lasting depolarization and increases in inpul resistance of dentate granule cells (Stanton et al., 1989) provides a mechanism that, if it occurs in CA 1 pyramidal neurons, could account for BAP.

Thus, we hypothesize that in the dentate gyrus there may be two distinct forms of NE-related plasticity occurring simultaneously; one action of NE would be at a presynaptic site to facilitate glutamate release, which would lead to a direct increase in the fEPSP response, and which would correspond to what has been termed NE-LLP. Previous studics have demonstrated via direct means that $\mathrm{NE}$ can enhance stimulated release of radiolabeled glutamate from both hippocampus (Lynch and Bliss, 1986) and cerebellum (Dolphin, 1982), consistent with a presynaptic action of NE. A second and perhaps completely unrelated effect of $\mathrm{NE}$ would be the prolonged increase in the population spike response observed in the CAl region that is not accompanied by corresponding changes in the fEPSP, and which is most likely the result of the persistent depolarization of the pyramidal neurons by ISO (Dunwiddie et al., 1991). This form of potentiation we refer to as BAP, and we hypothesize that a similar process may occur in the dentate gyrus in addition to the presynaptic effects of ISO. These results suggest that there are at least two discrete forms of NE-related long-lasting plasticity occurring in the hippocampus: NE-LLP in the dentate gyrus, which primarily involves a potentiation of glutamatergic transmission, and BAP in the CA1, and possibly dentate, as well. The NE modulation of LTP that occurs in both the dentate gyrus and in CA3, but not CA1 (Hopkins and Johnston, 1988; Stanton and Sarvey, 1985b), may represent yet another form of related plasticity.

The hippocampus has often been implicaled in memory formation. Until now, there has been no evidence to suggest that NE plays a role in long-term plasticity in the CAl region. Our own studies have demonstrated that lesions of noradrenergic inputs to $\mathrm{CA} 1$, depletions of $\mathrm{NE}$, and noradrenergic agonists and antagonists do not appear to antagonize LTP in the CA1 region (Dunwiddie et al., 1982), and this has been confirmed in other laboratories, as well (Stanton and Sarvey, 1985b). The present study demonstrates that NE can induce a long-term increase in the excitability of the pyramidal neurons in the CAI region. If this is the result of a persistent depolarization of the pyramidal neurons, then this would facilitate the induction of LTP in this brain region by helping to remove the voltagedependent block of the NMDA receptor-operated channel that is critical to the initiation of LTP in this brain region. Thus, though the changes induced by NE may be only relatively persistent (i.e., hours, rather than days or weeks), NE may produce a transitory "state" in which more enduring changes in function can be facilitated by intermediate-term changes induced by $\beta$-adrenoceptor activation.

\section{References}

Anwyl R, Rowan MJ (1984) Neurophysiological evidence for tricyclic antidepressant-induced beta-adrenergic responsiveness in the rat hippocampus. Brain Res 300:192-194.

Basile AS, Dunwiddie TV (1984) Norepinephrine elicits both excitatory and inhibitory responses from Purkinje cells in the in vitro rat cerebellar slice. Brain Res 296:15-25.

Bliss TVP, Lomo T (1973) Long-lasting potentiation of synaptic transmission in the dentate area of the anaesthetized rabbit following stimulation of the perforant path. J Physiol (Lond) 232:331-356.

Bliss TVP, Goddard GV, Riives M (1983) Reduction of long-term potentiation in the dentate gyrus of the rat following selective depletion of monoamines. J Physiol (Lond) 334:475-491.

Burgard EC, Decker G, Sarvey JM (1989) NMDA receptor antagonists block norepinephrine-induced long-lasting potentiation and long-term potentiation in rat dentate gyrus. Brain Res 482:351-355.

Dolphin AC (1982) Noradrenergic modulation of glutamate release in the cerebellum. Brain Res 252:111-116.

Dunwiddie TV, Lynch GS (1978) Long-term potentiation and depression of synaptic responses in the rat hippocampus: localization and frequency dependency. J Physiol (Lond) 276:353-367.

Dunwiddie TV, Mueller AL, Palmer MR, Stewart JM, Hoffer BJ (1980) Electrophysiological interactions of enkephalins with neuronal circuitry in the rat hippocampus. I. Effects on pyramidal cell activity. Brain Res 184:311-330.

Dunwiddie TV, Roberson NL, Worth TS (1982) Modulation of longterm potentiation: effects of adrenergic and neuroleptic drugs. Pharmacol Biochem Behav 17:1257-1264.

Dunwiddie TV, Taylor M, Heginbotham LR, Proctor WR (1991) Cellular mechanisms underlying long-term increases in the evoked population spike in the CAl region of rat hippocampus induced by $\beta$-adrenergic stimulation. $J$ Neurosci, in press.

Fowler JC, O'Donnell JM (1988) Antagonism of the responses to isoproterenol in the rat hippocampal slice with subtype-selective antagonists. Eur J Pharmacol 153:105-110. 
Haas HL, Konnerth A (1983) Histamine and noradrenaline decrease calcium-activated potassium conductance in hippocampal pyramidal cells. Nature 302:432-434.

Hopkins WF, Johnston D (1988) Noradrenergic enhancement of longterm potentiation at mossy fiber synapses in the hippocampus. $J$ Neurophysiol 59:667-687.

Lacaille JC, Harley CW (1985) The action of norepinephrine in the dentate gyrus: beta-mediated facilitation of evoked potentials in vitro. Brain Res 358:210-220.

Loy R, Koziell DA, Lindsey JD, Moore RY (1980) Noradrenergic innervation of the adult rat hippocampal formation. J Comp Neurol 189:699-710.

Lynch MA, Bliss TVP (1986) Noradrenaline modulates the release of $\left[{ }^{14} \mathrm{C}\right]$ glutamate from dentate but not from $\mathrm{CA} 1 / \mathrm{CA} 2$ slices of rat hippocampus. Neuropharmacology 25:493-498.

Madison DV, Nicoll RA (1982) Noradrenaline blocks accommodation of pyramidal cell discharge in the hippocampus. Nature 299:636-638.

Madison DV, Nicoll RA (1986a) Actions of noradrenaline recorded intracellularly in rat hippocampal CAl pyramidal neurones in vitro. J Physiol (Lond) 372:221-224.

Madison DV, Nicoll RA (1986b) Cyclic adenosine 3',5'-monophosphate mediates $\beta$-receptor actions of noradrenaline in rat hippocampal pyramidal cells. J Physiol (Lond) 372:245-259.

Moises HC, Woodward DJ, Hoffer BJ, Freedman R (1979) Interactions of norepinephrine with Purkinje cell responses to putative amino acid neurotransmitters applied by microiontophoresis. Exp Neurol 64:493-515.

Mueller AL, Hoffer BJ, Dunwiddie TV (1981) Noradrenergic responses in rat hippocampus: evidence for mediation by alpha and beta receptors in the in vitro slice. Brain Res 214:113-126.

Mueller AL, Kirk KL, Hoffer BJ, Dunwiddie TV (1982a) Noradrenergic responses in rat hippocampus: electrophysiological actions of direct- and indirect-acting sympathomimetics in the in vitro slice. $\mathbf{J}$ Pharmacol Exp Ther 223:599-605.

Mueller AL, Palmer MR, Hoffer BJ, Dunwiddie TV (1982b) Ilippocampal noradrenergic responses in vivo and in vitro. Characterization of alpha and beta components. Naunyn Schmiedebergs Arch Pharmacol 318:259-266.

Mynlieff M, Dunwiddie TV (1988) Noradrenergic depression of synaptic responses in hippocampus of rat: evidence for mediation by alpha ${ }_{1}$-receptors. Neuropharmacology 27:391-398.

Neuman RS, Harley CW (1983) Long-lasting potentiation of the dentate gyrus population spike by norepinephrine. Brain Res 273:162165.

Rainbow TC, Parsons B, Wolfe BB (1984) Quantitative autoradiography of $\alpha_{1}$-and $\alpha_{2}$-adrenergic receptors in rat brain. Proc Natl Acad Sci USA 81:1585-1589.
Rogawski MA, Aghajanian GK (1980) Modulation of lateral geniculate neurone excitability by noradrenaline microiontophoresis or locus coeruleus stimulation. Nature 287:731-734.

Schwartzkroin PA, Wester K (1975) Long-lasting facilitation of a synaptic potential following tetanization in the in vitro hippocampal slice. Brain Res 89:107-119.

Scgal M (1982) Norcpincphrine modulates reactivity of hippocampal cells to chemical stimulation in vitro. Exp Neurol 77:86-93.

Segal M, Bloom FE (1974a) The action of norepinephrine in the rat hippocampus. I. Iontophoretic studies. Brain Res 72:79-97.

Segal M, Bloom FE (1974b) The action of norepinephrine in the rat hippocampus. II. Activation of the input pathway. Brain Res 72:99114.

Smith CB, Moises HC, Spengler RN, Hollingsworth PJ (1989) Changes

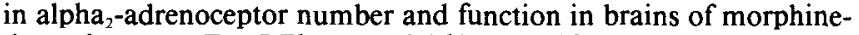
dependent rats. Eur J Pharmacol 161:111-119.

Stanton PK, Sarvey JM (1985a) Blockade of norepinephrine-induced long-lasting potentiation in the hippocampal dentate gyrus by an inhibitor of protein synthesis. Brain Res 361:276-283.

Stanton PK, Sarvey JM (1985b) Depletion of norepinephrine, but not scrotonin, reduccs long-term potentiation in the dentate gyrus of rat hippocampal slices. J Neurosci 5:2169-2176.

Stanton PK, Sarvey JM (1987) Norepinephrine regulates long-term potentiation of both the population spike and dendritic EPSP in hippocampal dentate gyrus. Brain Res Bull 18:115-119.

Stanton PK, Mody I, Heinemann U (1989) A role for $N$-methyl-Daspartate receptors in norepinephrine-induced long-lasting potentiation in the dentate gyrus. Exp Brain Res 77:517-530.

Su MT, Dunwiddie TV, Gerhardt GA (1990) Combined electrochemical and electrophysiological studies of monoamine overflow in rat hippocampal slice. Brain Res 518:149-158.

Washburn M, Moises HC (1989) Electrophysiological correlates of presynaptic alpha $a_{2}$-receptor-mediated inhibition of norepinephrine release at locus coeruleus synapses in dentate gyrus. J Neurosci 9: 2131-2140.

Waterhouse BD, Sessler FM, Cheng JT, Woodward DJ, Azizi SA, Moises HC (1988) New evidence for a gating action of norepinephrine in central neuronal circuits of mammalian brain. Brain Res Bull 21 : $425-432$.

Woodward DJ, Moises HC, Waterhouse BD, Hoffer BJ, Freedman R (1979) Modulatory actions of norepinephrine in the central nervous system. Fed Proc 38:2109-2116.

Yasuda RP, Zahniser NR, Dunwiddie TV (1984) Electrophysiological effects of cocaine in the rat hippocampus in vitro. Neurosci Lett 45 : 199-204. 